\title{
Helioseismology space and ground based studies
}

\author{
R. I. Kostik ${ }^{1}$, S. N. Osipov ${ }^{1}$, E. V. Khomenko ${ }^{1}$ \\ and N. I. Lebedev ${ }^{2}$ \\ ${ }^{1}$ Main Astronomical Observatory, National Academy of Sciences, 03680, Kyiv, Ukraine email: \\ kostik@mao.kiev.ua \\ ${ }^{2}$ IZMIRAN, Russian Academy of Sciences, Troitsk, Russia email: lebedev@rssi.izmiran.ru
}

\begin{abstract}
This is a preliminary report on the observations of solar irradiance fluctuations with the DIFOS photometer aboard the Russian-Ukrainian satellite CORONAS-F launched in 2001. In addition the parallel ground-based spectral observations (VTT, Tenerife) carried out with the first 20-days observing space campaign are described.
\end{abstract}

The Russian-Ukrainian satellite CORONAS-F has been launched on July 31, 2001. The orbit is quasi-synchronous with height $500-550 \mathrm{~km}$ and period 95 minutes. Among experiments aboard there is the photometer DIFOS-F. The design performs the continuous registration of solar irradiance within six spectral bands (range 350-1600 nm) with high flux resolution. Real measurements of the solar irradiance started on August 22 . The CORONAS-F provides 20-days of continuous observing time without gaps by sunset. The observed data is reducing now. The sets of power spectra of solar atmosphere have been obtained. They demonstrate the increasing of power with height (figure 1).

There was a possibility to coordinate the space observations aboard the CORONAS-F and the ground-base observations obtained with high spatial resolution. The latter observations were carried out on August 20-26, 2001 simultaneously with the first 20-days observing campaign of DIFOS photometer. The ground based instrument was $70 \mathrm{~cm}$ German Vacuum Tower Telescope (VTT) of the Observatorio del Teide (Instituto de Astrofísica de Canarias). The photospheric velocity and intensity oscillations of the different patterns of the solar surface were recorded with high spatial (0.5 arcsec) and temporal $(9.3 \mathrm{sec})$ resolution. We used a time series of CCD spectrograms in the range from 30 min to 120 min. The CCD camera, with $1024 \times 1024$ photosensitive elements, collected the spectral data. The observed images contained the Fe I $532.4 \mathrm{~nm}$ and $639.3 \mathrm{~nm}$ spectral lines with good height coverage from the low photosphere up to the temperature minimum region.

The similar data was obtained using SOHO-MDI experiment. The study of acoustic waves propagation through granulation patterns has been carried out. Both the ground based and space data (figure 2) testify the increase of oscillation amplitudes over continuum contrast.

\section{Acknowledgements}

This work has been partially supported by the Ukrainian Foundation for Pure Researches (project 02.07/00044) and by the INTAS project 00-00084. 


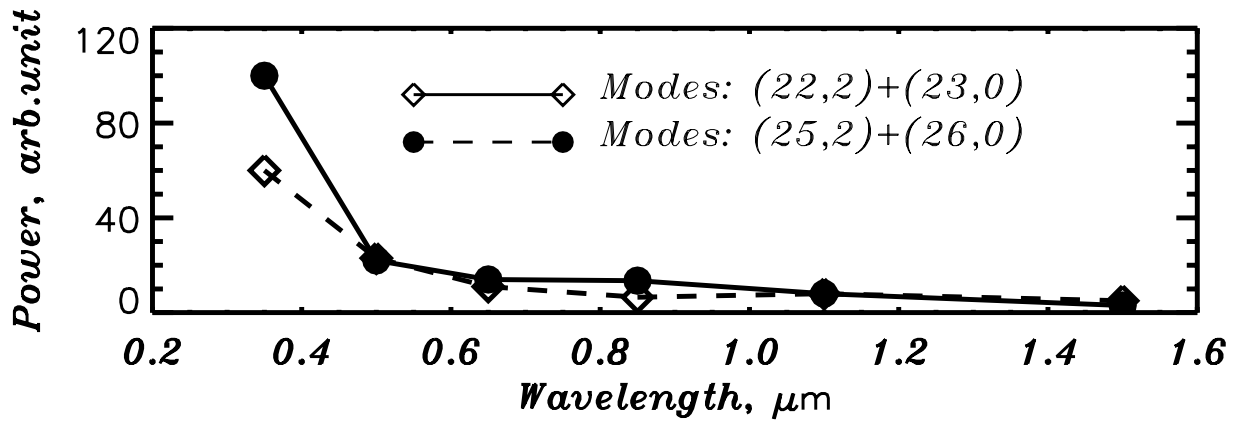

Figure 1. The power of individual modes in different channels.
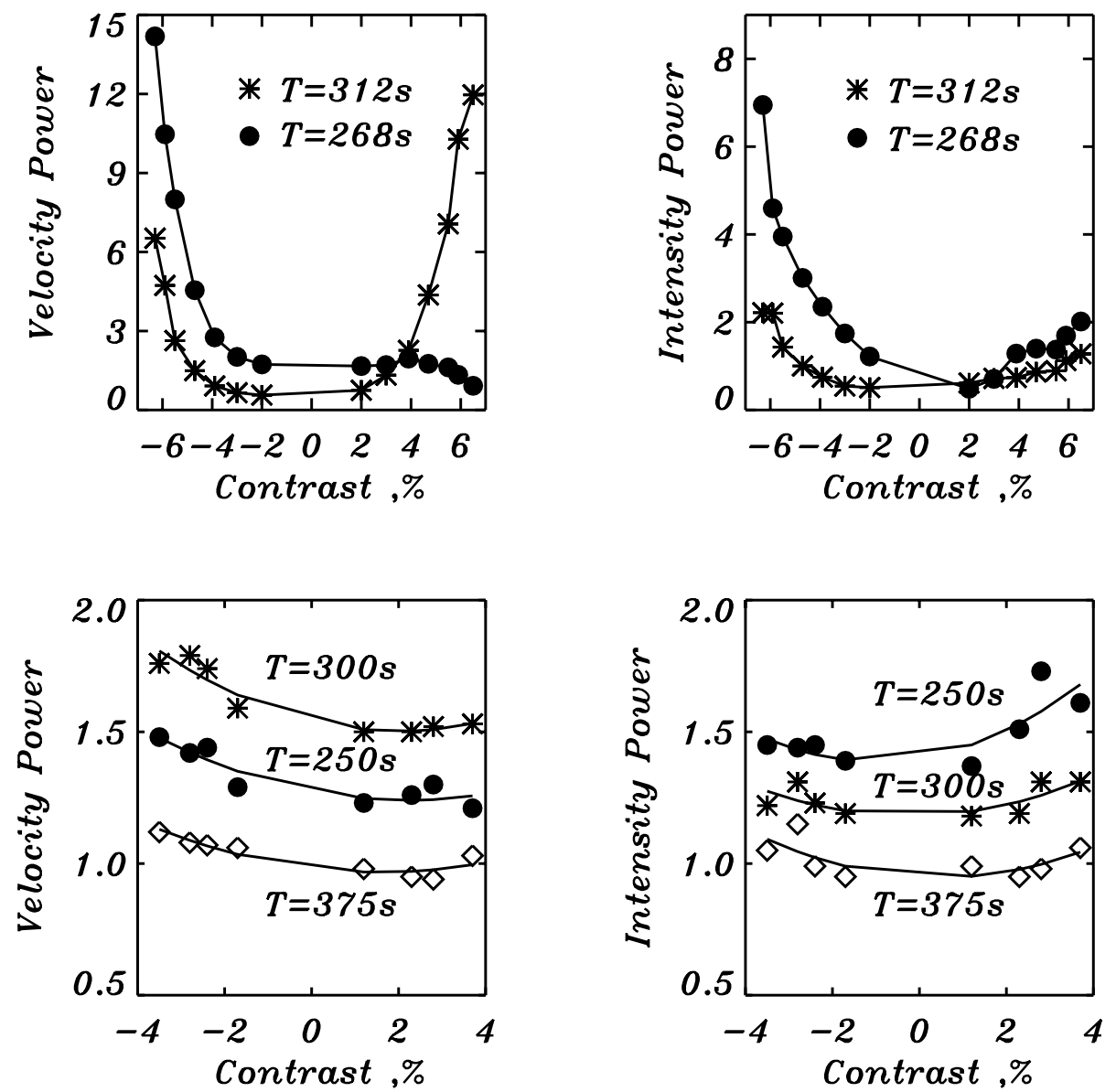

Figure 2. The power of the velocity and intensity oscillations over granules and intergranular lanes from VTT data for two periods (upper two panels) and from SOHO-MDI data for three periods (bottom two panels). 